\title{
IONIC LIQUID-ACCELERATED SYNTHESIS OF SOME N-ALKYL DERIVATIVES OF PHTHALIMIDE AND SULFONAMIDES
}

\author{
ALIREZA HASANINEJAD ${ }^{4 *}$, ABDOLKARIM ZARE ${ }^{* *}$, ALI KHALAFI-NEZHAD ${ }^{C}$, HASHEM SHARGHI \\ AHMAD REZA MOOSAVIZARE ${ }^{C}$, ABOLFATH PARHAMIC
}

\author{
${ }^{a}$ Department of Chemistry, Faculty of Sciences, Persian Gulf University, Bushehr 75169, Iran \\ ${ }^{b}$ Department of Chemistry, Payame Noor University of Bushehr, Bushehr 1698, Iran \\ ${ }^{c}$ Department of Chemistry, College of Sciences, Shiraz University, Shiraz 71454, Iran \\ (Received: October 3, 2007 - Accepted: July 14, 2008)
}

\begin{abstract}
Ionic liquid 1-butyl-3-methylimidazolium bromide ([bmim]Br) efficiently accelerates Michael addition of phthalimide and sulfonamides to $\alpha, \beta$-unsaturated esters in the presence of $\mathrm{K}_{2} \mathrm{CO}_{3}$ under microwave irradiation (MW) to afford $N$-alkyl derivatives of these compounds in high yields and short reaction times.
\end{abstract}

Keywords: Ionic liquid; $N$-Alkyl phthalimide; $N$-Alkyl sulfonamide; Michael addition; Microwave.

\section{INTRODUCTION}

The exploitation of ionic liquids as solvents in organic transformations has been reported extensively during the past decade..$^{1-5}$ The most useful properties of ionic liquids are the ability to dissolve a wide range of substances, very low vapor pressure, high thermal stability, and the fact that they can be stored for long times without decomposition. Moreover, it is often possible to achieve reactions in ionic liquids that otherwise proceed with great difficulty, or even not at all. ${ }^{1-3}$ Together with the substitution of common molecular solvents, nonconventional activation methods have been applied as powerful tools to decrease reaction times and to enhance reactivity, mainly microwave irradiation. ${ }^{6-13}$

Michael addition reaction has been used in the carbon-carbon, ${ }^{14,15}$ carbonoxygen, ${ }^{16}$ carbon-sulfur, ${ }^{17,18}$ and carbon-nitrogen bonds formation. ${ }^{19-23}$ AzaMichael addition of phthalimide and sulfonamides to $\alpha, \beta$-unsaturated esters provides a direct and appealing route toward synthesis of $\mathrm{N}$-alkyl derivatives of these compounds. ${ }^{24-27}$ For $N$-alkyl phthalimides some biological activities, such as antipsychotic, ${ }^{28}$ anti-inflammatory, ${ }^{29,30}$ hypolipidemic, ${ }^{31}$ and receptor properties have been reported. ${ }^{32}$ Furthermore, these compounds are very useful intermediate in organic synthesis as they can be easily converted to primary amines (Gabriel synthesis). ${ }^{33} \mathrm{~N}$-Alkylated sulfonamides have also various biological activities, including antidepressant, psychostimulant, analgesic, anti-ulcer, anti-emetic anti-inflammatory properties. ${ }^{34-37}$ Moreover, saccharin as a sulfonamide derivative and its derivatives are very important in medicinal chemistry. ${ }^{36,37}$ To the best of our knowledge, the preparation of $\mathrm{N}$-alkyl derivatives of phthalimide and sulfonamides by aza-conjugate addition reaction has been scarcely studied in the literature. Moe et al. have used $\mathrm{Na}$ in absolute EtOH to achieve Michael addition of phthalimide to $\alpha, \beta$-unsaturated aldehydes. ${ }^{24}$ Cardillo et al. have carried out the reaction of phthalimide salts to $\alpha, \beta$-unsaturated imides in the presence of $\mathrm{AlMe}_{2} \mathrm{Cl}^{25}$ For Michael addition of sulfonamides to $\alpha, \beta$-unsaturated compounds, $\mathrm{Al}_{2} \mathrm{O}_{3},{ }^{26}$ and $\mathrm{PBu}_{3}{ }^{27}$ has been employed. Furthermore, more recently we have performed this transformation using $\mathrm{K}_{2} \mathrm{CO}_{3}$ /tetrabutylammonium bromide (TBAB). As mentioned, $\mathrm{K}_{2} \mathrm{CO}_{3}$ accompanied with $\mathrm{TBAB}$ has been used previously only for Michael reaction of sulfonamides not phthalimide and saccharin. Moreover, in this report, sulfonamides have been $N$-alkylated with moderate selectivity in the synthesis of $\mathrm{N}$-alkyl and $\mathrm{N}, \mathrm{N}$-dialkyl sulfonamides. ${ }^{38}$ The mentioned methods are also associated with one or more of the following drawbacks: moderate yields, relatively long reaction times and the use of more reactive Michael acceptors. Because of wide range of biological and synthetic applications of $N$-alkyl phthalimides and sulfonamides, their preparation has received renewed interest processes.

Potassium carbonate $\left(\mathrm{K}_{2} \mathrm{CO}_{3}\right)$ is a cheap, commercially available, reusable and green base which has been used frequently in $N-,{ }^{39-42} C-, 43,44$ and $O$ alkylation reactions..$^{45-47}$

Having the above aspects in mind and also in continuation of our previous studies on aza-Michael reactions, ${ }^{48,49}$ green organic synthesis, ${ }^{39,48-57}$ as well as applications of $\mathrm{K}_{2} \mathrm{CO}_{3}$ in $\mathrm{N}$-alkylation reactions, ${ }^{39}$ we report here our results on the synthesis of $N$-alkyl derivatives of phthalimide and sulfonamides via Michael addition of phthalimide and sulfonamides to $\alpha, \beta$-unsaturated esters using $\mathrm{K}_{2} \mathrm{CO}_{3}$ in [bmim] Br under microwave irradiation (Schemes 1 and 2).

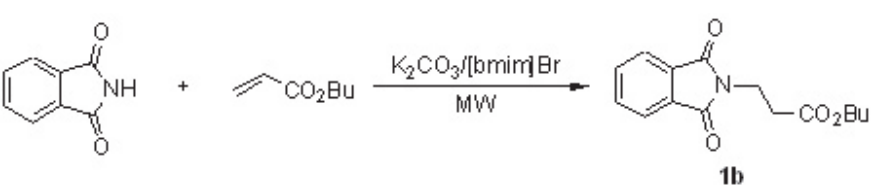

Scheme 1.

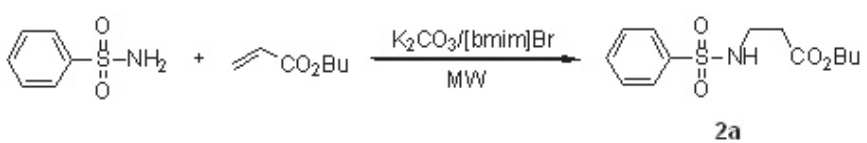

Scheme 2.

\section{RESULTS AND DISCUSSION}

We have found previously $\mathrm{K}_{2} \mathrm{CO}_{3}$ acts as an efficient reagent for highly regioselective $N$-alkylation of benzotriazole. ${ }^{39}$ Moreover, this reagent has been frequently applied in alkylation reactions. ${ }^{40-47}$ These subjects encouraged us to use this reagent for $\mathrm{N}$-alkylation of phthalimide and sulfonamides via Michael reaction. Thus, at first, we used $\mathrm{K}_{2} \mathrm{CO}_{3}$ to accomplish Michael addition of phthalimide to $n$-butyl acrylate as a model reaction under microwave conditions in [bmim $] \mathrm{Br}$ (Scheme 1). Interestingly, the reaction proceeded efficiently at $300 \mathrm{~W}$ of microwave power (max. $130^{\circ} \mathrm{C}$ ) and the desired Michael adduct $\mathbf{1 b}$ was obtained in excellent yield in short reaction time (Table 1). We also studied the influence of different bases to evaluate their capabilities (Table 1). As it is shown in Table 1, the best results were obtained when $\mathrm{K}_{2} \mathrm{CO}_{3}$ was applied as base. We extended this reaction to benzenesulfonamide as Michael donor that provided compound $\mathbf{2 a}$ in $85 \%$ yield within $6 \mathrm{~min}$ (Scheme 2). Considering the excellent results obtained from $\mathrm{K}_{2} \mathrm{CO}_{3}$, this base was used for all reactions.

Table 1. The effect of various bases on Michael addition of phthalimide $(2 \mathrm{mmol})$ to $n$-butyl acrylate $(2.4 \mathrm{mmol})$ in [bmim] $\mathrm{Br}(1 \mathrm{~g})$ under microwave conditions $\left(300 \mathrm{~W}, \max .130^{\circ} \mathrm{C}\right)$

\begin{tabular}{|l|l|l|l|}
\hline Entry & Base & Time (min) & Yield $^{a}(\%)$ \\
\hline 1 & $\mathrm{~K}_{2} \mathrm{CO}_{3}(0.25$ eq. $)$ & 15 & 59 \\
\hline 2 & $\mathrm{~K}_{2} \mathrm{CO}_{3}(0.5$ eq. $)$ & 10 & 74 \\
\hline 3 & $\mathrm{~K}_{2} \mathrm{CO}_{3}(1$ eq. $)$ & 7 & 96 \\
\hline 4 & $\mathrm{Na}_{2} \mathrm{CO}_{3}(1$ eq. $)$ & 12 & 76 \\
\hline 5 & $t$-BuOK (1 eq.) & 12 & 80 \\
\hline 6 & $\mathrm{CaO}(1$ eq. $)$ & 15 & 47 \\
\hline 7 & $\mathrm{MgO}(1$ eq. $)$ & 15 & 39 \\
\hline 8 & $\mathrm{NBu}_{3}(1$ eq. $)$ & 12 & 84 \\
\hline
\end{tabular}

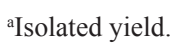


$N$-alkylation of phthalimide with $n$-butyl acrylate was also studied in several ionic liquids, such as $[\mathrm{bmim}] \mathrm{Br},[\mathrm{bmim}] \mathrm{Cl},[\mathrm{bmim}] \mathrm{BF}_{4}$ and $[\mathrm{bmim}] \mathrm{PF}_{6}$ using $\mathrm{K}_{2} \mathrm{CO}_{3}$ under microwave irradiation. Higher yields and shorter reaction times were observed in $[\mathrm{bmim}] \mathrm{Br}$ and $[\mathrm{bmim}] \mathrm{Cl}$. However, [bmim] $\mathrm{Br}$ was applied as solvent for all reactions, because, the preparation of this ionic liquid was easier in comparison with the others.

In order to determine whether ionic liquid was an essential factor to promote the reaction, the model reaction was carried out in several traditional solvents, and the results are depicted in Table 2. As Table 2 indicates, longer reaction times were required in these classical solvents than those in ionic liquid to achieve the reaction. Furthermore, the yields were not so high as those in ionic liquid. It was clear that the ionic liquids accelerated this reaction.

Table 2. Comparative Michael addition of phthalimide $(2 \mathrm{mmol})$ to $n$ butyl acrylate $(2.4 \mathrm{mmol})$ in the presence of $\mathrm{K}_{2} \mathrm{CO}_{3}(2 \mathrm{mmol})$ in conventional solvents $(5 \mathrm{~mL})$ versus [bmim] $\mathrm{Br}(1 \mathrm{~g})$ in microwave conditions $(300 \mathrm{~W}$, max. $\left.130^{\circ} \mathrm{C}\right)$

\begin{tabular}{|l|l|l|l|}
\hline Entry & Solvent & Time (min) & Yield $^{a}(\%)$ \\
\hline 1 & - & 25 & 23 \\
\hline 2 & DMSO & 20 & 63 \\
\hline 3 & DMF & 20 & 55 \\
\hline 4 & HMPTA & 20 & 32 \\
\hline 5 & {$[$ bmim $] \mathrm{Br}$} & 7 & 96 \\
\hline
\end{tabular}

asolated yield.

To establish the efficiency and applicability of this method, we introduced phthalimide and sulfonamides to different $\alpha, \beta$-unsaturated esters. The results are summarized in Tables 3, 4 and 5). As Tables 3, 4 and 5 shows, the reactions proceeded efficiently and the desired Michael adducts were obtained in good to excellent yields and short reaction times.

It has been observed that the bulkiness of alkoxy group (-OR) of $\alpha, \beta-$ unsaturated esters did not affect significantly on the yields and the reaction times (Table 3, Table 4: entries 1 and 2 as well as Table 5). Michael addition of phthalimide and saccharin to substituted $\alpha, \beta$-unsaturated esters ethyl methacrylate and ethyl crotonate was not efficient; however, sulfonamides reacted successfully with these esters (Table 4: entries 3 and 4). Lower reaction yield was obtained when 4-methylbenzenesulfonamides instead of benzenesulfonamides was applied in the reaction (Table 4: entry 5). Saccharin afforded lower yields of products in comparison to the other Michael donors (Table 5). We tried to improve the yields of $N$-alkyl derivatives of saccharin; however, our attempt was not successful.

Table 3. Michael addition of phthalimide $(2 \mathrm{mmol})$ to $\alpha, \beta$-unsaturated esters (2.4 mmol) using $\mathrm{K}_{2} \mathrm{CO}_{3}(2 \mathrm{mmol})$ in [ $\left.\mathrm{bmim}\right] \mathrm{Br}(1 \mathrm{~g})$ under microwave irradiation $\left(300 \mathrm{~W}, \max .130^{\circ} \mathrm{C}\right)$

\begin{tabular}{|c|l|c|c|c|}
\hline \multicolumn{3}{|l|}{} \\
Entry & $\mathrm{R}$ & Product & $\begin{array}{c}\text { Time } \\
(\mathrm{min})\end{array}$ & $\begin{array}{c}\mathrm{Yield}^{\mathrm{a}} \\
(\%)\end{array}$ \\
\hline 1 & $\mathrm{CH}_{3} \mathrm{CH}_{2}$ & $\mathbf{1 a}$ & 7 & 94 \\
\hline 2 & $\mathrm{CH}_{3}\left(\mathrm{CH}_{2}\right)_{2} \mathrm{CH}_{2}$ & $\mathbf{1 b}$ & 7 & 96 \\
\hline 3 & $\mathrm{CH}_{3}\left(\mathrm{CH}_{2}\right)_{4} \mathrm{CH}_{2}$ & $\mathbf{1 c}$ & 7 & 95 \\
\hline 4 & $\mathrm{C}_{6} \mathrm{H}_{5} \mathrm{CH}_{2}$ & $\mathbf{1 d}$ & 7 & 95 \\
\hline 5 & $\mathrm{C}_{6} \mathrm{H}_{5} \mathrm{CH}_{2} \mathrm{CH}_{2}$ & $\mathbf{1 e}$ & 8 & 94 \\
\hline 6 & $\mathrm{C}_{6} \mathrm{H}_{5} \mathrm{CH}_{2} \mathrm{CHCH}_{2}$ & $\mathbf{1 f}$ & 8 & 93 \\
\hline 7 & ${ }_{0}-\mathrm{CH}_{3} \mathrm{O}_{-} \mathrm{C}_{6} \mathrm{H}_{4} \mathrm{OCH}_{2} \mathrm{CH}(\mathrm{OH}) \mathrm{CH}_{2}$ & $\mathbf{1 g}$ & 10 & 90 \\
\hline 8 & $\mathrm{C}_{6} \mathrm{H}_{5}$ & $\mathbf{1 h}$ & 5 & 91 \\
\hline
\end{tabular}

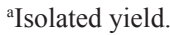

Table 4. Michael addition of sulfonamides $(2 \mathrm{mmol})$ to $\alpha, \beta$-unsaturated esters $(2.2 \mathrm{mmol})$ using $\mathrm{K}_{2} \mathrm{CO}_{3}(2 \mathrm{mmol})$ in $[\mathrm{bmim}] \mathrm{Br}(1 \mathrm{~g})$ under microwave irradiation $\left(300 \mathrm{~W}, \max .110^{\circ} \mathrm{C}\right)$

\begin{tabular}{|c|c|c|c|c|c|c|c|}
\hline & $\mathrm{J}_{0}^{\circ}-\mathrm{NH}_{2}+$ & & $\mathrm{CO}_{2} \mathrm{R}^{3}$ & $\frac{\mathrm{K}_{2} \mathrm{CO}_{3} \pi \mathrm{bm} \text { in }}{\mathrm{MW}}$ & Ar & $\mathrm{NH}_{\mathrm{C}}^{\mathrm{R}}$ & \\
\hline & ${ }^{1}, R^{2}=H_{1} M e$ & $R^{3}=A \mid$ & & & & $2 a-2 e$ & \\
\hline Entry & $\mathrm{Ar}$ & $\mathrm{R}^{1}$ & $\mathrm{R}^{2}$ & $\mathrm{R}^{3}$ & Product $^{b}$ & $\begin{array}{l}\text { Time } \\
(\mathrm{min})\end{array}$ & $\begin{array}{l}\text { Yield }^{a} \\
(\%)\end{array}$ \\
\hline 1 & $\mathrm{C}_{6} \mathrm{H}_{5}$ & $\mathrm{H}$ & $\mathrm{H}$ & $\begin{array}{l}\mathrm{CH}_{3}\left(\mathrm{CH}_{2}\right)_{2} \\
\mathrm{CH}_{2}\end{array}$ & $2 a$ & 6 & 85 \\
\hline 2 & $\mathrm{C}_{6} \mathrm{H}_{5}$ & $\mathrm{H}$ & $\mathrm{H}$ & $\begin{array}{l}\mathrm{C}_{6} \mathrm{H}_{5} \mathrm{CH}= \\
\mathrm{CHCH}_{2}\end{array}$ & $2 b$ & 7 & 83 \\
\hline 3 & $\mathrm{C}_{6} \mathrm{H}_{5}$ & $\mathrm{H}$ & $\mathrm{CH}_{3}$ & $\mathrm{CH}_{3} \mathrm{CH}_{2}$ & $2 c$ & 10 & 79 \\
\hline 4 & $\mathrm{C}_{6} \mathrm{H}_{5}$ & $\mathrm{CH}_{3}$ & $\mathrm{H}$ & $\mathrm{CH}_{3} \mathrm{CH}_{2}$ & $2 d$ & 14 & 74 \\
\hline 5 & $\begin{array}{l}p- \\
\mathrm{CH}_{3} \mathrm{C}_{6} \mathrm{H}_{4}\end{array}$ & $\mathrm{H}$ & $\mathrm{H}$ & $\begin{array}{l}\mathrm{CH}_{3}\left(\mathrm{CH}_{2}\right)_{2} \\
\mathrm{CH}_{2}\end{array}$ & $2 e$ & 8 & 78 \\
\hline
\end{tabular}

a Isolated yield. 'In this method, the selectivity was high and $\mathrm{N}, \mathrm{N}$ dialkylated sulfonamides were produced beside $N$-alkylated products in low yields $(7-14 \%)$.

Table 5. Michael addition of saccharin (as a sulfonamide derivative) (2 mmol) to $\alpha, \beta$-unsaturated esters $(3 \mathrm{mmol})$ using $\mathrm{K}_{2} \mathrm{CO}_{3}(2 \mathrm{mmol})$ in $[\mathrm{bmim}] \mathrm{Br}$ $(1 \mathrm{~g})$ under microwave irradiation $\left(200 \mathrm{~W}\right.$, max. $\left.100^{\circ} \mathrm{C}\right)$

\begin{tabular}{|c|c|c|c|c|}
\hline \multicolumn{2}{|c|}{$R=A \mid k y l$} & \multicolumn{3}{|c|}{$3 a-3 g$} \\
\hline Entry & $\mathrm{R}$ & Product & $\begin{array}{l}\text { Time } \\
\text { (min) }\end{array}$ & $\begin{array}{c}\text { Yield }^{a} \\
(\%)\end{array}$ \\
\hline 1 & $\mathrm{CH}_{3}\left(\mathrm{CH}_{2}\right)_{2} \mathrm{CH}_{2}$ & $3 \mathbf{a}$ & 2 & 42 \\
\hline 2 & $\mathrm{CH}_{3}\left(\mathrm{CH}_{2}\right)_{4} \mathrm{CH}_{2}$ & $3 \mathbf{b}$ & 2 & 41 \\
\hline 3 & $\mathrm{C}_{6} \mathrm{H}_{5} \mathrm{CH}_{2}$ & $3 c$ & 2 & 43 \\
\hline 4 & $\mathrm{C}_{6} \mathrm{H}_{5} \mathrm{CH}=\mathrm{CHCH}_{2}$ & 3d & 2 & 40 \\
\hline 5 & $o-\mathrm{CH}_{3} \mathrm{O}-\mathrm{C}_{6} \mathrm{H}_{4} \mathrm{OCH}_{2} \mathrm{CH}(\mathrm{OH}) \mathrm{CH}_{2}$ & $3 e$ & 2 & 37 \\
\hline 6 & $\left(\mathrm{CH}_{3}\right)_{2} \mathrm{CH}$ & 3f & 2 & 41 \\
\hline 7 & Cyclohexyl & $3 g$ & 2 & 39 \\
\hline
\end{tabular}

asolated yield.

Ease of recycling of the reagent is one of the most advantages of our method. For the reaction of phthalimide with $n$-butyl acrylate no significant loss of the product yield was observed when $\mathrm{K}_{2} \mathrm{CO}_{3} /[\mathrm{bmim}] \mathrm{Br}$ was used after five times recycling.

\section{CONCLUSIONS}

In summary, we have developed an efficient method for Michael addition of phthalimide and sulfonamides to $\alpha, \beta$-unsaturated esters. This new strategy for the synthesis of $N$-Alkyl phthalimides and sulfonamides as biologically interesting compounds has some advantages, including high yield, short reaction time, ease of product isolation, low cast, potential for recycling of ionic liquid as well as reagent and compliance with green chemistry protocols

\section{EXPERIMENTAL}

All chemicals were purchased from Merck or Fluka chemical companies. All reactions were carried out using laboratory microwave oven (MW 3000, Landgraf Company, Germany). IR spectra were run on a Shimadzu FTIR-8300 
spectrophotometer. The ${ }^{1} \mathrm{H}$ NMR $(250 \mathrm{MHz})$ and ${ }^{13} \mathrm{C}$ NMR $(62.5 \mathrm{MHz})$ were run on a Bruker Avanced DPX-250, FT-NMR spectrometer. Mass spectra were recorded on a Shimadzu GC MS-QP 1000 EX apparatus. Melting points were recorded on a Büchi B-545 apparatus in open capillary tubes.

General procedure for Michael addition of phthalimide and sulfonamides to $\alpha, \beta$-unsaturated esters

To a mixture of compounds consisting of phthalimide or sulfonamide (2 mmol), well-ground $\mathrm{K}_{2} \mathrm{CO}_{3}(2 \mathrm{mmol})$ and $\alpha, \beta$-unsaturated ester ( 2.2 to $3 \mathrm{mmol}$, Tables 3, 4 and 5) in a microwave vessel was added [bmim] $\operatorname{Br}(1 \mathrm{~g})$ and mixed carefully. The resulting mixture was irradiated in a microwave oven for the powers, the temperatures and the times reported in Tables 3,4 and 5. Afterward, the reaction mixture was cooled to room temperature and was extracted with $\mathrm{Et}_{2} \mathrm{O}(3 \times 50 \mathrm{~mL})$. The organic extracts were then combined. After removal of the solvent, the crude product was purified by column chromatography on silica gel eluted with EtOAc/ $n$-hexane (1/5 for $N$-alkyl phthalimides and saccharins and $1 / 3$ for $N$-alkyl sulfonamides). After isolation of the products and evaporating of the remainder $\mathrm{Et}_{2} \mathrm{O}$ in ionic liquid, the ionic liquid containing reagent $\mathrm{K}_{2} \mathrm{CO}_{3}$ $\left(\mathrm{K}_{2} \mathrm{CO}_{3} /[\mathrm{bmim}] \mathrm{Br}\right)$ was used for next run under identical reaction conditions.

Ethyl 3-phthalimido propanoate (1a)

Colorless solid; $\mathrm{mp} 60-61{ }^{\circ} \mathrm{C}$; IR (KBr): 3051, 2968, 1774, $1716 \mathrm{~cm}^{-1}$; ${ }^{1} \mathrm{H}$ NMR $\left(\mathrm{CDCl}_{3}\right): \delta 1.11\left(3 \mathrm{H}, \mathrm{t}, J=7.1 \mathrm{~Hz}, \mathrm{CH}_{3}\right), 2.57(2 \mathrm{H}, \mathrm{t}, J=7.2 \mathrm{~Hz}$, $\left.\mathrm{O}=\mathrm{CCH}_{2}\right), 3.86\left(2 \mathrm{H}, \mathrm{t}, J=7.2 \mathrm{~Hz}, \mathrm{NCH}_{2}\right), 4.06\left(2 \mathrm{H}, \mathrm{q}, J=7.1 \mathrm{~Hz}, \mathrm{OCH}_{2}\right)$, 7.57-7.70 $(4 \mathrm{H}, \mathrm{m}) ;{ }^{13} \mathrm{C}$ NMR $\left(\mathrm{CDCl}_{3}\right): \delta 13.8,32.7,33.5,64.3,122.9$ (two carbons), 131.7 (two carbons), 133.8 (two carbons), 167.5 (two carbons), 170.6; $\operatorname{MS}(\mathrm{m} / \mathrm{z}): 247\left(\mathrm{M}^{+}\right)$

Butyl 3-phthalimido propanoate (1b)

Colorless solid; mp 51-52 ${ }^{\circ} \mathrm{C}$; IR (KBr): $3069,2960,1774,1716 \mathrm{~cm}^{-1} ;{ }^{1} \mathrm{H}$ $\operatorname{NMR}\left(\mathrm{CDCl}_{3}\right): \delta 0.90\left(3 \mathrm{H}, \mathrm{t}, J=6.8 \mathrm{~Hz}, \mathrm{CH}_{3}\right), 1.28\left(2 \mathrm{H}, \mathrm{m}, \mathrm{CH}_{3} \mathrm{CH}_{2}\right), 1.59$ $\left(2 \mathrm{H}, \mathrm{m}, \mathrm{CH}_{3} \mathrm{CH}_{2} \mathrm{CH}_{2}\right), 2.58\left(2 \mathrm{H}, \mathrm{t}, J=7.2 \mathrm{~Hz}, \mathrm{O}=\mathrm{CCH}_{2}\right), 3.91(2 \mathrm{H}, \mathrm{t}, J=7.2 \mathrm{~Hz}$, $\left.\mathrm{NCH}_{2}\right), 4.08(2 \mathrm{H}, \mathrm{t}, J=6.9 \mathrm{~Hz}, \mathrm{OCH}), 7.64-7.76(4 \mathrm{H}, \mathrm{m}) ;{ }^{13} \mathrm{C} \mathrm{NMR}\left(\mathrm{CDCl}_{3}\right)$ : $\delta 13.3,18.5,30.2,32.5,33.4,64.1,122.8$ (two carbons), 131.7 (two carbons), 133.6 (two carbons), 167.3 (two carbons), 170.3; MS (m/z): $275\left(\mathrm{M}^{+}\right)$.

Hexyl 3-phthalimido propanoate (1c)

Colorless solid; mp 40-41 ${ }^{\circ} \mathrm{C}$; IR (KBr): 3055, 2970, 1774, $1716 \mathrm{~cm}^{-1}$; ${ }^{1} \mathrm{H}$ NMR $\left(\mathrm{CDCl}_{3}\right): \delta 0.89\left(3 \mathrm{H}, \mathrm{t}, J=6.7 \mathrm{~Hz}, \mathrm{CH}_{3}\right), 1.29-1.36(6 \mathrm{H}, \mathrm{m}), 1.60$ $\left(2 \mathrm{H}, \mathrm{m}, \mathrm{CH}_{3}\left(\mathrm{CH}_{2}\right)_{3} \mathrm{CH}_{2}\right), 2.59\left(2 \mathrm{H}, \mathrm{t}, J=7.0 \mathrm{~Hz}, \mathrm{O}=\mathrm{CCH}_{2}\right), 3.95(2 \mathrm{H}, \mathrm{t}, J=$ $7.0 \mathrm{~Hz}, \mathrm{NCH}), 4.09(2 \mathrm{H}, \mathrm{t}, J=6.9 \mathrm{~Hz}, \mathrm{OCH}), 7.68-7.83(4 \mathrm{H}, \mathrm{m}) ;{ }^{13} \mathrm{C} \mathrm{NMR}$ $\left(\mathrm{CDCl}_{3}\right): \delta 13.7,22.1,25.4,28.1,30.2,32.4 .33 .4,64.3,122.7$ (two carbons), 132.1 (two carbons), 133.6 (two carbons), 167.2 (two carbons), 170.2; MS ( $\mathrm{m} /$ z): $303\left(\mathrm{M}^{+}\right)$

Benzyl 3-phthalimido propanoate (1d)

Colorless solid; mp 57-59 ${ }^{\circ} \mathrm{C}$; IR (KBr): $3061,2958,1774,1716 \mathrm{~cm}^{-1} ;{ }^{1} \mathrm{H}$ $\operatorname{NMR}\left(\mathrm{CDCl}_{3}\right): \delta 2.59\left(2 \mathrm{H}, \mathrm{t}, J=7.1 \mathrm{~Hz}, \mathrm{O}=\mathrm{CCH}_{2}\right), 3.86(2 \mathrm{H}, \mathrm{t}, J=7.1 \mathrm{~Hz}$, $\left.\mathrm{NCH}_{2}\right), 4.94(2 \mathrm{H}, \mathrm{s}, \mathrm{OCH}), 7.08-7.17(5 \mathrm{H}, \mathrm{m}), 7.52(2 \mathrm{H}, \mathrm{m}), 7.63(2 \mathrm{H}, \mathrm{m}) ;{ }^{13} \mathrm{C}$ $\operatorname{NMR}\left(\mathrm{CDCl}_{3}\right): \delta 32.9,33.7,66.6,122.6$ (two carbons), 128.2 (two carbons), 128.5, 129.4 (two carbons), 131.9 (two carbons), 133.9 (two carbons), 135.6, 167.8 (two carbons), 170.6; MS (m/z): $309\left(\mathrm{M}^{+}\right)$.

Phenethyl 3-phthalimido propanoate (1e)

Colorless solid; mp 53-55 ${ }^{\circ} \mathrm{C}$; IR (KBr): $3052,2933,1774,1716 \mathrm{~cm}^{-1} ;{ }^{1} \mathrm{H}$ $\operatorname{NMR}\left(\mathrm{CDCl}_{3}\right): \delta 2.69(2 \mathrm{H}, \mathrm{t}, J=7.2 \mathrm{~Hz}, \mathrm{O}=\mathrm{CCH}), 2.87(2 \mathrm{H}, \mathrm{t}, J=7.1 \mathrm{~Hz}$, $\mathrm{ArCH}), 3.93\left(2 \mathrm{H}, \mathrm{t}, J=7.2 \mathrm{~Hz}, \mathrm{NCH}_{2}\right), 4.26\left(2 \mathrm{H}, \mathrm{t}, J=7.1 \mathrm{~Hz}, \mathrm{OCH}_{2}\right), 7.13-$ $7.26(5 \mathrm{H}, \mathrm{m}), 7.64(2 \mathrm{H}, \mathrm{m}), 7.77(2 \mathrm{H}, \mathrm{m}) ;{ }^{13} \mathrm{C} \mathrm{NMR}\left(\mathrm{CDCl}_{3}\right): \delta 32.8,33.6,34.9$, 65.2, 123.1 (two carbons), 126.4, 128.3 (two carbons), 128.9 (two carbons), 132.7 (two carbons), 133.9 (two carbons), 137.6, 167.8 (two carbons), 170.7; MS $(\mathrm{m} / \mathrm{z}): 323\left(\mathrm{M}^{+}\right)$.

\section{Cinnamyl 3-phthalimido propanoate (1f)}

Pale yellow oil; IR (neat): 3061, 2954, 1774, 1716, $1497 \mathrm{~cm}^{-1} ;{ }^{1} \mathrm{H}$ NMR $\left(\mathrm{CDCl}_{3}\right): \delta 2.68\left(2 \mathrm{H}, \mathrm{t}, J=7.1 \mathrm{~Hz}, \mathrm{O}=\mathrm{CCH}_{2}\right), 3.92\left(2 \mathrm{H}, \mathrm{t}, J=7.1 \mathrm{~Hz}, \mathrm{NCH}_{2}\right)$, $4.64(2 \mathrm{H}, \mathrm{m}, \mathrm{OCH}), 6.12(1 \mathrm{H}, \mathrm{m}, \mathrm{ArCH}=C H), 6.49(1 \mathrm{H}, \mathrm{d}, J=15.8 \mathrm{~Hz}$, $\mathrm{ArCH}), 7.17-7.26(5 \mathrm{H}, \mathrm{m}), 7.57(2 \mathrm{H}, \mathrm{m}), 7.72(2 \mathrm{H}, \mathrm{m}) ;{ }^{13} \mathrm{C} \mathrm{NMR}\left(\mathrm{CDCl}_{1}\right): \delta$ $32.9,33.7,65.4,122.8$ (two carbons), 123.2, 126.6 (two carbons), 128.0, 128.5 (two carbons), 131.9 (two carbons), 133.9 (two carbons), 134.4, 136.1, 167.9 (two carbons), 170.6; $\mathrm{MS}(\mathrm{m} / \mathrm{z}): 335\left(\mathrm{M}^{+}\right)$

2-Hydroxy-3-(2-methoxyphenoxy)propyl 3-phthalimido propanoate (1g) Pale yellow oil; IR (neat): 3480, 3049, 2948, 1770, $1715 \mathrm{~cm}^{-1} ;{ }^{1} \mathrm{H}$ NMR $\left(\mathrm{CDCl}_{3}\right): \delta 2.63\left(2 \mathrm{H}, \mathrm{t}, J=7.0 \mathrm{~Hz}, \mathrm{O}=\mathrm{CCH}_{2}\right), 3.67\left(3 \mathrm{H}, \mathrm{s}, \mathrm{CH}_{3}\right), 3.80-390(5 \mathrm{H}$, m), 4.11-4.19 $(3 \mathrm{H}, \mathrm{m}), 6.71-6.79(4 \mathrm{H}, \mathrm{m}), 7.54(2 \mathrm{H}, \mathrm{m}), 7.68(2 \mathrm{H}, \mathrm{m}) ;{ }^{13} \mathrm{C}$ $\operatorname{NMR}\left(\mathrm{CDCl}_{3}\right): \delta 32.8,33.6,55.7,65.7,67.9,70.5,111.9,114.2,120.9,121.8$ (two carbons), 123.2, 131.7 (two carbons), 134.0 (two carbons), 147.8, 149.3, 168.0 (two carbons), 170.8; MS (m/z): $399\left(\mathrm{M}^{+}\right)$.

Phenyl 3-phthalimido propanoate (1h)

Colorless solid; mp 70-72 ${ }^{\circ} \mathrm{C}$; IR (KBr): 3076, 2970, 1775, $1713 \mathrm{~cm}^{-1} ;{ }^{1} \mathrm{H}$
NMR $\left(\mathrm{CDCl}_{3}\right): \delta 2.74\left(2 \mathrm{H}, \mathrm{t}, J=7.1 \mathrm{~Hz}, \mathrm{O}=\mathrm{CCH}_{2}\right), 3.95(2 \mathrm{H}, \mathrm{t}, J=7.1 \mathrm{~Hz}$, $\mathrm{NCH}), 6.98(2 \mathrm{H}, \mathrm{d}, J=8.0 \mathrm{~Hz}), 7.09(1 \mathrm{H}, \mathrm{d}, J=6.9), 7.24(2 \mathrm{H}, \mathrm{dd}, J=6.9,8.0$ $\mathrm{Hz}), 7.61-7.73(4 \mathrm{H}, \mathrm{m}) ;{ }^{13} \mathrm{C} \mathrm{NMR}\left(\mathrm{CDCl}_{3}\right): \delta 32.9,33.5,121.4$ (two carbons), 122.9 (two carbons), 125.7, 129.2 (two carbons), 131.6 (two carbons), 133.9 (two carbons), 150.3, 167.5 (two carbons), 170.3; MS ( $\mathrm{m} / \mathrm{z}): 295\left(\mathrm{M}^{+}\right)$.

Butyl 3-(phenylsulfonamido)propanoate (2a)

Colorless oil (Lit. ${ }^{38}$ oil); IR (neat): 3271, 3048, 2960, 1733, 1447, 1330 $\mathrm{cm}^{-1} ;{ }^{1} \mathrm{H} \mathrm{NMR}\left(\mathrm{CDCl}_{3}\right): \delta 0.90\left(3 \mathrm{H}, \mathrm{t}, J=6.5 \mathrm{~Hz}, \mathrm{CH}_{3}\right), 1.34\left(2 \mathrm{H}, \mathrm{m}, \mathrm{CH}_{3} \mathrm{CH}_{2}\right)$, $1.56\left(2 \mathrm{H}, \mathrm{m}, \mathrm{CH}_{2} \mathrm{CH}_{2} \mathrm{CH}_{2}\right), 2.51\left(2 \mathrm{H}, \mathrm{t}, J=5.0 \mathrm{~Hz}, \mathrm{O}=\mathrm{CCH}_{2}\right), 3.19 \mathrm{t}, 2 \mathrm{H}$, $\left.J=5.0 \mathrm{~Hz}, \mathrm{O}=\mathrm{CCCH}_{2} \mathrm{CH}_{2}\right), 4.03\left(\mathrm{t}, 2 \mathrm{H}, J=7.0 \mathrm{~Hz}, \mathrm{OCH}_{2}\right), 5.68(1 \mathrm{H}, \mathrm{s}, \mathrm{NH}$, exchangeable with $\left.\mathrm{D}_{2} \mathrm{O}\right), 7.48-7.57\left(3 \mathrm{H}\right.$, complex, $\mathrm{H}_{3}-\mathrm{H}_{5}$ of aromatic ring), $7.79\left(2 \mathrm{H}, \mathrm{m}, \mathrm{H}_{2}\right.$ and $\mathrm{H}_{6}$ of aromatic ring $) ;{ }^{13} \mathrm{C} \mathrm{NMR}\left(\mathrm{CDCl}_{3}\right): \delta 13.5,18.9,30.4$, $34.1,38.7,64.6,126.8,129.0$ (two carbons), 132.5 (two carbons), 139.9, 172.7; $\operatorname{MS}(\mathrm{m} / \mathrm{z}): 285\left(\mathrm{M}^{+}\right)$

\section{Cinnamyl 3-(phenylsulfonamido)propanoate (2b)}

Pale yellow oil (Lit. ${ }^{38}$ oil); IR (neat): 3287, 3059, 2964, 1732, 1447, 132 $9 \mathrm{~cm}^{-1} ;{ }^{1} \mathrm{H}$ NMR $\left(\mathrm{CDCl}_{3}\right): \delta 2.55\left(2 \mathrm{H}, \mathrm{t}, J=5.2 \mathrm{~Hz}, \mathrm{O}=\mathrm{CCH}_{2}\right), 3.19(2 \mathrm{H}, \mathrm{t}, J$ $\left.=5.2 \mathrm{~Hz}, \mathrm{O}=\mathrm{CCH}_{2} \mathrm{CH}_{2}\right), 4.69\left(2 \mathrm{H}, \mathrm{m}, \mathrm{OCH}_{2}\right), 5.50(1 \mathrm{H}, \mathrm{s}, \mathrm{NH}), 6.23(1 \mathrm{H}, \mathrm{m}$, $\mathrm{PhCH}=C H), 6.63(1 \mathrm{H}, \mathrm{d}, J=15.7 \mathrm{~Hz}, \mathrm{PhCH}), 7.29-7.35\left(5 \mathrm{H}\right.$, complex, $\mathrm{H}_{1}-\mathrm{H}_{5}$ of aromatic ring of alkoxy group), 7.45-7.50 (3H, complex, $\mathrm{H}_{3} \mathrm{H}_{5}$ of aromatic ring of sulfonamide), $7.95\left(2 \mathrm{H}, \mathrm{m}, \mathrm{H}_{2}\right.$ and $\mathrm{H}_{6}$ of aromatic ring of sulfonamide); ${ }^{13} \mathrm{C} \mathrm{NMR}\left(\mathrm{CDCl}_{3}\right): \delta 34.2,38.8,65.8,122.6,122.9,126.6$ (two carbons), 126.9, 128.1, 128.6 (two carbons), 129.0 (two carbons), 132.7 (two carbons), 134.6, 139.9, 171.7; MS (m/z): $345\left(\mathrm{M}^{+}\right)$

Ethyl 2-methyl-3-(phenylsulfonamido)propanoate (2c)

Pale yellow oil (Lit. ${ }^{38}$ oil); IR (neat): 3283, 3034, 2966, 1732, 1447, $1328 \mathrm{~cm}^{-1} ;{ }^{1} \mathrm{H}$ NMR $\left(\mathrm{CDCl}_{3}\right): \delta 1.17-1.26\left(6 \mathrm{H}\right.$, complex, $\left.2 \mathrm{CH}_{3}\right), 2.73(1 \mathrm{H}, \mathrm{m}$, $\mathrm{O}=\mathrm{CCH}), 3.14-3.20\left(2 \mathrm{H}\right.$, complex, $\left.\mathrm{O}=\mathrm{CCH}\left(\mathrm{CH}_{3}\right) \mathrm{CH}_{2}\right), 4.12(2 \mathrm{H}, \mathrm{q}, J=7.0$ $\left.\mathrm{Hz}, \mathrm{OCH}_{2}\right), 5.65(1 \mathrm{H}, \mathrm{s}, \mathrm{NH}), 7.55-7.64\left(3 \mathrm{H}\right.$, complex, $\mathrm{H}_{3}-\mathrm{H}_{5}$ of aromatic ring), $7.93\left(2 \mathrm{H}, \mathrm{m}, \mathrm{H}_{2}\right.$ and $\mathrm{H}_{6}$ of aromatic ring); ${ }^{13} \mathrm{C} \mathrm{NMR}\left(\mathrm{CDCl}_{3}\right): \delta 14.0,14.7,39.6$, 45.4, 60.8, 126.8, 129.1 (two carbons), 132.5 (two carbons), 139.9, 174.7; MS $(\mathrm{m} / \mathrm{z}): 271\left(\mathrm{M}^{+}\right)$.

Ethyl 3-(phenylsulfonamido)butanoate (2d)

Pale yellow solid; mp $60-62{ }^{\circ} \mathrm{C}\left(\mathrm{Lit}^{38} \mathrm{mp} 60-62{ }^{\circ} \mathrm{C}\right)$; IR (KBr): 3285,3044 , 2953, 1732, 1447, $1329 \mathrm{~cm}^{-1} ;{ }^{1} \mathrm{H}$ NMR $\left(\mathrm{CDCl}_{3}\right): \delta$ 1.14-1.23 (6H, complex, $\left.2 \mathrm{CH}_{3}\right), 2.38-2.43\left(2 \mathrm{H}\right.$, complex, $\left.\mathrm{O}=\mathrm{CCH}_{2}\right), 3.68\left(1 \mathrm{H}, \mathrm{m}, \mathrm{O}=\mathrm{CCH}_{2} \mathrm{CH}\right), 4.04$ $\left(2 \mathrm{H}, \mathrm{q}, J=7.1 \mathrm{~Hz}, \mathrm{OCH}_{2}\right), 5.39(1 \mathrm{H}, \mathrm{s}, \mathrm{NH}), 7.49-7.59\left(3 \mathrm{H}\right.$, complex, $\mathrm{H}_{3}-\mathrm{H}_{5}$ of aromatic ring), $7.89\left(2 \mathrm{H}, \mathrm{m}, \mathrm{H}_{2}\right.$ and $\mathrm{H}_{6}$ of aromatic ring); ${ }^{13} \mathrm{C} \mathrm{NMR}\left(\mathrm{CDCl}_{3}\right): \delta$ $14.0,21.0,40.7,46.6,60.7,126.9,129.0$ (two carbons), 132.5 (two carbons), 140.9, 171.1; MS $(\mathrm{m} / \mathrm{z}): 271\left(\mathrm{M}^{+}\right)$.

Butyl 3-(4-methylphenylsulfonamido)propanoate (2e)

Pale yellow oil (Lit. ${ }^{38}$ oil); IR (neat): 3286, 3044, 2960, 1732, $1330 \mathrm{~cm}^{-1}$; ${ }^{1} \mathrm{H}$ NMR $\left(\mathrm{CDCl}_{3}\right): \delta 0.89\left(3 \mathrm{H}, \mathrm{t}, J=6.8 \mathrm{~Hz}, \mathrm{CH}_{2}\left(\mathrm{CH}_{2}\right)_{2} \mathrm{CH}_{3}\right), 1.33(2 \mathrm{H}, \mathrm{m}$, $\left.\mathrm{CH}_{3} \mathrm{CH}_{2}\right), 1.56\left(2 \mathrm{H}, \mathrm{m}, \mathrm{CH}_{3} \mathrm{CH}_{2} \mathrm{CH}_{2}\right), 2.40(3 \mathrm{H}, \mathrm{s}, \mathrm{ArCH}), 2.51(2 \mathrm{H}, \mathrm{t}, J=$ $5.3 \mathrm{~Hz}, \mathrm{O}=\mathrm{CCH}), 3.16\left(2 \mathrm{H}, \mathrm{t}, J=5.3 \mathrm{~Hz}, \mathrm{O}=\mathrm{CCH}_{2} \mathrm{CH}_{2}\right), 4.04(2 \mathrm{H}, \mathrm{t}, J=7.0$ $\left.\mathrm{Hz}, \mathrm{OCH}_{2}\right), 5.76(1 \mathrm{H}, \mathrm{s}, N H), 7.33\left(2 \mathrm{H}, \mathrm{d}, J=7.9 \mathrm{~Hz}, \mathrm{H}_{3}\right.$ and $\mathrm{H}_{5}$ of aromatic ring), $7.72\left(2 \mathrm{H}, \mathrm{d}, J=7.9 \mathrm{~Hz}, \mathrm{H}_{2}\right.$ and $\mathrm{H}_{6}$ of aromatic ring $) ;{ }^{33} \mathrm{C} \mathrm{NMR}^{5}\left(\mathrm{CDCl}_{3}\right): \delta$ $13.5,18.9,21.3,30.3,34.1,38.7,64.5,126.9,129.6$ (two carbons), 135.8 (two carbons), 143.2, 172.7; MS (m/z): $299\left(\mathrm{M}^{+}\right)$.

(3a)

Butyl 3-(1,1-dioxido-3-oxo-1,2-benzisothiazol-2(3H)-yl) propanoate

Yellow oil; IR (neat): 3039, 2938, 1733, 1455, $1333 \mathrm{~cm}^{-1} ;{ }^{1} \mathrm{H}$ NMR $\left(\mathrm{CDCl}_{3}\right): \delta 0.84(3 \mathrm{H}, \mathrm{t}, J=7.4 \mathrm{~Hz}, \mathrm{CH}), 1.25\left(2 \mathrm{H}, \mathrm{m}, \mathrm{CH}_{3} \mathrm{CH}_{2}\right), 1.51(2 \mathrm{H}$ $\left.\mathrm{m}, \mathrm{CH}_{3} \mathrm{CH}_{2} \mathrm{CH}_{2}\right), 2.80\left(2 \mathrm{H}, \mathrm{t}, J=6.8 \mathrm{~Hz}, \mathrm{O}=\mathrm{CCH}_{2}\right), 4.00-4.08(4 \mathrm{H}, \mathrm{m}, \mathrm{NCH}$ and $\left.\mathrm{OCH}_{2}\right), 7.81-7.97(4 \mathrm{H}, \mathrm{m}) ;{ }^{13} \mathrm{C}$ NMR $\left(\mathrm{CDCl}_{3}\right): \delta 12.6,18.0,29.4,32.0$, $33.6,63.9,119.9,124.2,126.2,133.3,133.8,136.4,157.6,169.3$; MS $(\mathrm{m} / \mathrm{z})$ : $311\left(\mathrm{M}^{+}\right)$. (3b)

Hexyl 3-(1,1-dioxido-3-oxo-1,2-benzisothiazol-2(3H)-yl)propanoate

Pale yellow oil; IR (neat): 3051, 2931, 1734, 1457, $1339 \mathrm{~cm}^{-1} ;{ }^{1} \mathrm{H}$ NMR $\left(\mathrm{CDCl}_{3}\right): \delta 0.78\left(3 \mathrm{H}, \mathrm{t}, J=7.0 \mathrm{~Hz}, \mathrm{CH}_{3}\right), 1.16-1.25(6 \mathrm{H}, \mathrm{m}), 1.53(2 \mathrm{H}, \mathrm{m}$, $\mathrm{CH}_{3}\left(\mathrm{CH}_{2}\right)_{2} \mathrm{CH}_{2}, 2.81(2 \mathrm{H}, \mathrm{t}, \mathrm{J}=6.7 \mathrm{~Hz}, \mathrm{O}=\mathrm{CCH}), 3.95-4.04(4 \mathrm{H}, \mathrm{m}, \mathrm{NCH}$ and $\left.\mathrm{OCH}_{2}\right), 7.78-7.92(4 \mathrm{H}, \mathrm{m}) ;{ }^{13} \mathrm{C}$ NMR $\left(\mathrm{CDCl}_{3}\right): \delta 12.9,21.4,24.4,27.4$, $30.3,32.0,33.6,64.1,119.9,124.1,126.1,133.4,133.9,136.6,157.6,169.2$; $\operatorname{MS}(\mathrm{m} / \mathrm{z}): 339\left(\mathrm{M}^{+}\right)$

Benzyl 3-(1,1-dioxido-3-oxo-1,2-benzisothiazol-2(3H)-yl)propanoate(3c)

Pale yellow oil; IR (neat): $3059,2947,1733,1339 \mathrm{~cm}^{-1} ;{ }^{1} \mathrm{H} \mathrm{NMR}\left(\mathrm{CDCl}_{3}\right)$ : $\delta 2.78\left(2 \mathrm{H}, \mathrm{t}, J=6.8 \mathrm{~Hz}, \mathrm{O}=\mathrm{CCH}_{2}\right), 3.99\left(2 \mathrm{H}, \mathrm{t}, J=6.8 \mathrm{~Hz}, \mathrm{NCH}_{2}\right), 5.02(2 \mathrm{H}, \mathrm{s}$, $\mathrm{OCH}), 7.11-7.21(5 \mathrm{H}, \mathrm{m}), 7.79-7.94(4 \mathrm{H}, \mathrm{m}):{ }^{13} \mathrm{C} \mathrm{NMR}(\mathrm{CDCl}): \delta 32.6,33.9$, $66.9,119.6,123.9,126.6,128.0$ (two carbons), 128.4, 129.7 (two carbons), 133.2, 133.7, 135.8, 136.2, 157.4, 169.1; MS $(\mathrm{m} / \mathrm{z}): 345\left(\mathrm{M}^{+}\right)$.

Cinnamyl 3-(1,1-dioxido-3-oxo-1,2-benzisothiazol-2(3H)-yl) propanoate (3d)

Yellow oil; IR (neat): 3061, 2954, 1733, $1338 \mathrm{~cm}^{-1} ;{ }^{1} \mathrm{H} \mathrm{NMR}\left(\mathrm{CDCl}_{3}\right): \delta$ 
$2.85\left(2 \mathrm{H}, \mathrm{t}, J=6.9 \mathrm{~Hz}, \mathrm{O}=\mathrm{CCH}_{2}\right), 4.03(2 \mathrm{H}, \mathrm{t}, J=6.9 \mathrm{~Hz}, \mathrm{NCH}), 4.70(2 \mathrm{H}, \mathrm{m}$, $\left.\mathrm{OCH}_{2}\right), 6.23(1 \mathrm{H}, \mathrm{m}, \mathrm{ArCH}=C H), 6.54(1 \mathrm{H}, \mathrm{d}, J=15.7 \mathrm{~Hz}, \mathrm{ArCH}), 7.19-7.30$ $(5 \mathrm{H}, \mathrm{m}), 7.76-7.93(4 \mathrm{H}, \mathrm{m}) ;{ }^{13} \mathrm{C} \mathrm{NMR}\left(\mathrm{CDCl}_{3}\right): \delta 32.1,33.6,64.5,119.9,121.7$, 124.2, 125.6, 126.1, 126.7 (two carbons), 127.0, 127.5 (two carbons), 133.3, 133.5, 133.8, 136.8, 157.5, 168.5; $\mathrm{MS} \mathrm{m} / \mathrm{z}(\%): 307\left(\mathrm{M}^{+}-\mathrm{SO}_{2}\right)$.

2-Hydroxy-3-(2-methoxyphenoxy)propyl 3-(1,1-dioxido-3-oxo-1,2benzisothiazol-2( $3 H$ )-yl)propanoate (3e)

Pale yellow oil; IR (neat): 3475, 3059, 2953, 1734, $1339 \mathrm{~cm}^{-1} ;{ }^{1} \mathrm{H}$ NMR $\left(\mathrm{CDCl}_{3}\right): \delta 2.80\left(2 \mathrm{H}, \mathrm{t}, J=6.8 \mathrm{~Hz}, \mathrm{O}=\mathrm{CCH}_{2}\right), 3.61\left(3 \mathrm{H}, \mathrm{s}, \mathrm{CH}_{3}\right), 3.87-3.99$ $(5 \mathrm{H}, \mathrm{m}), 4.14-4.23(3 \mathrm{H}, \mathrm{m}), 6.68-6.83(4 \mathrm{H}, \mathrm{m}), 7.80-7.94(4 \mathrm{H}, \mathrm{m}) ;{ }^{13} \mathrm{C} \mathrm{NMR}$ $\left(\mathrm{CDCl}_{3}\right): \delta 32.5,33.8,56.2,65.5,67.4,71.1,112.2,114.5,120.0,121.1,122.9$, $124.4,126.0,133.4,133.6,136.2,147.5,149.2,157.8,168.3 ; \mathrm{MS}(\mathrm{m} / \mathrm{z}): 371$ $\left(\mathrm{M}^{+}-\mathrm{SO}_{2}\right)$.

(3f)

Isopropyl3-(1,1-dioxido-3-oxo-1,2-benzisothiazol-2(3H)yl)propanoate

Pale yellow oil; IR (neat): 3033, 2982, 1734, 1457, $1340 \mathrm{~cm}^{-1} ;{ }^{1} \mathrm{H}$ NMR $\left(\mathrm{CDCl}_{3}\right): \delta 1.18\left(6 \mathrm{H}, \mathrm{d}, J=6.6 \mathrm{~Hz}, 2 \mathrm{CH}_{3}\right), 2.77\left(2 \mathrm{H}, \mathrm{t}, J=6.7, \mathrm{O}=\mathrm{CCH}_{2}\right), 4.01$ $\left(2 \mathrm{H}, \mathrm{t}, J=6.7 \mathrm{~Hz}, \mathrm{NCH}_{2}\right), 4.97(1 \mathrm{H}, \mathrm{q}, J=6.6, \mathrm{OCH}), 7.79-7.95(4 \mathrm{H}, \mathrm{m}) ;{ }^{13} \mathrm{C}$ NMR $\left(\mathrm{CDCl}_{3}\right): \delta 20.7$ (two carbons), 32.2, 33.6, 67.5, 119.9, 124.2, 126.2, 133.3, 133.8, 136.6, 157.6, 168.7; MS $(\mathrm{m} / \mathrm{z}): 297\left(\mathrm{M}^{+}\right)$.

Cyclohexyl 3-(1,1-dioxido-3-oxo-1,2-benzisothiazol-2(3H) yl) propanoate (3g)

Colorless solid; mp $62-64{ }^{\circ} \mathrm{C}$; IR (neat): $2938,1733,1339 \mathrm{~cm}^{-1} ;{ }^{1} \mathrm{H}$ NMR $\left(\mathrm{CDCl}_{3}\right): \delta 1.19-1.36(6 \mathrm{H}, \mathrm{m}), 1.65-1.81(4 \mathrm{H}, \mathrm{m}), 2.81(2 \mathrm{H}, \mathrm{t}, J=6.8 \mathrm{~Hz}$ $\left.\mathrm{O}=\mathrm{CCH}_{2}\right), 4.04\left(2 \mathrm{H}, \mathrm{t}, J=6.8 \mathrm{~Hz}, \mathrm{NCH}_{2}\right), 4.76(1 \mathrm{H}, \mathrm{m}, \mathrm{OCH}), 7.81-8.00(4 \mathrm{H}$ $\mathrm{m}) ;{ }^{13} \mathrm{C}$ NMR $\left(\mathrm{CDCl}_{3}\right.$ ): $\delta 23.6$ (two carbons), 25.2, 31.4, 33.2, 34.6 (two carbons), $73.3,120.9,125.1,127.1,134.3,134.8,137.5,158.6,169.6$; MS $(\mathrm{m} / \mathrm{z})$ : $273\left(\mathrm{M}^{+}-\mathrm{SO}_{2}\right)$.

\section{ACKNOWLEDGEMENTS}

We appreciate Payame Nour University of Bushehr, Persian Gulf University and Shiraz University research councils for financial support of this work.

\section{REFERENCES}

1. P. Wasserscheid, T. Welton, Ionic Liquids in Synthesis, Wiley-VCH, Weinheim, 2003

2. K. Mikami, Green Reaction Media in Organic Synthesis, Blackwell Publishing, UK, Oxford, 2005.

3. R. D. Rogers, Ionic Liquids As Green Solvents: Progress and Prospects, Publisher: An American Chemical Society Publication, 2005.

4. M. M. Khodaei, A. R. Khosropour, S. Ghaderi, J. Iran. Chem. Soc. 3, 69 , (2006).

5. M. M. Mojtahedi, M. S. Abaee, H. Abbasi, J. Iran. Chem. Soc. 3, 93, (2006).

6. A. Loupy, Microwaves in organic synthesis, Wiley-VCH, Weinheim, 2006.

7. K. Tanaka, Solvent-free organic synthesis, Wiley- $\mathrm{VCH}, \mathrm{GmbH}$ and $\mathrm{KGaA}$, Weinheim, 2004

8. R. S. Varma, Advances in green chemistry: chemical synthesis using microwave irradiation, Astra Zeneca Research Foundation, Kavitha Printers, Bangalore, India, 2002.

9. A. R. Khosropour, K. Esmaeilpoor, A. Moradie, J. Iran. Chem. Soc. 3, 81, (2006).

10. M. Mamaghani, K. Tabatabaeian, M. Mirzaeinejad, M. Nikpassand, $J$. Iran. Chem. Soc. 3, 89, (2006).

11. K. G. Desai, J. P. Raval, K. R. Desai, J. Iran. Chem. Soc. 3, 233, (2006).

12. S. Balalaie, M. Soleiman-Beigi, F. Rominger, J. Iran. Chem. Soc. 2, 319 , (2005).

13. H. Loghmani-Khouzani, M.M. Sadeghi, R. Ranjbar-Karimi, J. Iran. Chem. Soc. 2, 330, (2005).

14. P. Kotrusz, S. Toma, Arkivoc v, 100, (2006).

15. J. Comelles, M. Moreno-Mañas, A. Vallribera, Arkivoc ix, 207, (2005).

16. Y. Hayashi, K. Nishimura, Chem. Lett. 296, (2002).

17. B. Movassagh, P. Shaygan, Arkivoc xii, 130, (2006).

18. H. Firouzabadi, N. Iranpoor, A. A. Jafari, Synlett 299, (2005).

19. H. Firouzabadi, N. Iranpoor, M. Jafarpour, A. Ghaderi, J. Mol. Catal. A.: Chemical 252, 150, (2006).

20. H. Firouzabadi, N. Iranpoor, F. Nowrouzi, Chem. Commun. 789, (2005).

21. H. Firouzabadi, N. Iranpoor, A. A. Jafari, Adv. Synth. Cat. 347, 655, (2005).
22. B. Tamami, A. Fadavi, M. Tamami, Iran. Polym. J. 15, 799, (2006); Chem. Abstr. 149820, (2007).

23. M. R. Saidi, R. S. Brown, F. Rajabi, J. Iran. Chem. Soc. 2, 300, (2005).

24. O. A. Moe, D. T. Warner, J. Am. Chem. Soc. 71, 1251, (1949).

25. G. Cardillo, A. D. Simone, L. Gentilucci, P. Sabatino, C. Tomasini, Tetrahedron Lett. 35, 5051, (1994).

26. A. Reitz, M. Verlander, M. Goodman, Tetrahedron Lett. 23, 751, (1982).

27. C. Gimbert, M. Moreno-Maňas, E. Pérez, A. Vallribera, Tetrahedron 63, 8305, (2007).

28. M. H. Norman, D. J. Minick, G. C. Rigdon, J. Med. Chem. 39, 149, (1996).

29. L. M. Lima, P. Castro, A. L. Machado, C. A. M. Fraga, C. Lugnier, V. L. G. de Moraesc, E. J. Barreiroa, Bioorg. Med. Chem. 10, 3067, (2002).

30. X. Collin, J. M. Robert, G. Wielgosz, G. Le Baut, N. Grimaud, J. Y. Petit, Eur. J. Med. Chem. 36, 639, (2001).

31. J. M. Chapman, G. H. Cocolas, I. H. Hall, J. Med. Chem. 26, 243, (1983).

32. A. Raasch, O. Scharfestein, C. Trankle, U. Holzgrabe, K. Mohr, J. Med. Chem. 45, 3809, (2002).

33. M. B. Smith, J. March, Advanced Organic Chemistry: Reactions, Mechanisms and Structures, $4^{\text {th }}$ ed., John Wiley \& sons, New York, 2001.

34. M. J. O'neil, A. Smith, T. E. Heckelman, F. Dudavari, Merck index: an encyclopedia of chemicals, drugs, and biologicals, $13^{\text {th }}$ ed., Merck: Whitehouse Station, NG, 2001.

35. L. Soler, V. Cerrada, M. P. Matía, J. L. Novella, J. Alvarez-Builla, Arkivoc iv, 312, (2007).

36. Ö. Güzel, A. Salman, Bioorg. Med. Chem. 14, 7804, (2006).

37. J. C. Filho, A. O. Santini, A. L. M. Nasser, Food chemistry 83, 297, (2003).

38. G. H. Imanzadeha, A. Zare, A. Khalafi-Nezhad, A. Hasaninejad, A. R. Moosavi Zare, A. Parhami, J. Iran. Chem. Soc. 4, 467, (2007).

39. A. Khalafi-Nezhad, A. Zare, A. Parhami, M. N. Soltani Rad, G. R. Nejabat, J. Iran. Chem. Soc. 4, 271, (2007).

40. D. Bogdal, Molecules, 333, (1999).

41. D. Bogdal, J. Pielichowski, A. Boron, Synlett 873, (1996).

42. Y.-L. Chen, H.-M. Hung, C.-M. Lu, K.-C. Li, C.-C. Tzeng, Bioorg. Med. Chem. 12, 6539, (2004)

43. C. Rabeyrin, C. Nguefack, D. Sinou, Tetrahedron Lett. 41, 7461, (2000).

44. B. Lygo, J. Crosby, J. A. Peterson, Tetrahedron Lett. 40, 8671, (1999).

45. B. K. Pchelka, A. Loupy, A. Petit, Tetrahedron 62, 10968, (2006).

46. A. Loupy, P. Pigeon, M. Ramdani, Tetrahedron 52, 6705, (1996).

47. D. Bogdal, J. Pielichowski, A. Boron, Synth. Commun. 28, 3029, (1998).

48. A. Zare, A. Hasaninejad, A. Khalafi-Nezhad, A. Parhami, A. R. Moosavi Zare, J. Iran Chem. Soc. 5, 100, (2008).

49. A. Khalafi-Nezhad, A. Zarea, M. N. Soltani Rad, B. Mokhtari, A. Parhami, Synthesis 419, (2005)

50. A. Hasaninejad, H. Sharghi, Phosphorus, Sulfur, and Silicon 182, 873, (2007).

51. A. Hasaninejad, A. Zare, J. Sulfur Chem. 28, 357, (2007).

52. A. Hasaninejad, A. Zare, H. Sharghi, M. Shekouhy, R. Khalifeh, A. Salimi Beni, A. R. Moosavi Zare, Can. J. Chem. 85, 416, (2007).

53. A. Khalafi-Nezhad, A. Zare, A. Parhami, M. N. Soltani Rad, G. R. Nejabat, Phosphorus, Sulfur, and Silicon 182, 657, (2007).

54. A. Hasaninejad, A. Zare, H. Sharghi, A. Khalafi-Nezhad, A. Parhami, A. R. Moosavi Zare, Phosphorus, Sulfur, and Silicon, In press.

55. A. Hasaninejad, A. Parhami, A Zare, A. Khalafi-Nezhad, A. Nasrolahi Shirazi, A. R. Moosavi Zare, Polish J. Chem. 82, 565, (2008).

56. A. Khalafi-Nezhad, A. Zare, A. Parhami, M. N. Soltani Rad, G. R. Nejabat, Synth. Commun. 36, 3549, (2006).

57. A. Khalafi-Nezhad, A. Parhami, M. N. Soltani Rad, A. Zarea, Tetrahedron Lett. 46, 6879, (2005). 